IEEE Instrumentation and Measurement

Technology Conference

Budapest, Hungary, May 21-23, 2001

\title{
Resonator Based Non-parametric Identification of Linear Systems
}

\author{
László Sujbert, Gábor Péceli and Gyula Simon \\ Dept. of Measurement and Information Systems, Budapest University of Technology and Economics, 1521 Budapest, Hungary. \\ Phone: +36 1 463-2057, fax: +36 1463-4112, e-mail: [sujbert,peceli,simon]@mit.bme.hu.
}

\begin{abstract}
A$ nonparametric identification method for linear systems is proposed. The identificaton is done via synchronized multisine measurements where the synchronization is ensured by a resonator based generator - observer pair. The advantage of the proposed structure is that it works as a fitter bank and provides the measurement results on-line. Exponential averaging is an option of the method and it requires no extra calculations. A further advantage is that the identification can be done over any frequency set without any loss of performance. Explicit formulas are given for noise suppression and settling time. The method is illustrated by a practical example.
\end{abstract}

Keywords - Non-parametric frequency domain identification, multisine, resonator based observer, on-line measurement

\section{INTRODUCTION}

Fourier analysis is a well-known method for non-parametric frequency domain identification of linear systems [1]. Frequency domain data are often inputs for parametric identification [2]. The utilization of multisine excitation provides the possibility of the elimination of the systematic errors like leakage and picket fence [3]. In most cases the output of the system is analyzed by the discrete Fourier transform (DFT), while the frequency domain parameters of the excitation are known in advance. The DFT is calculated via the fast Fourier transform (FFT). In order to suppress the measurement noise, averaging on the analysis results is also necessary.

Resonator based observers were developed earlier to perform the recursive discrete Fourier transform (RDFT) [4], [5]. In these observers the resonators work in a common feedback loop providing zero steady-state feedback error at the resonator frequencies. The summed output of such resonators can generate any periodic signal up to the half of the sampling frequency. It is straightforward to utilize such a generator - observer pair for frequency domain non-parametric system identification: the system to be identified has to be in between the generator and the observer and the ratio of the state variables of the observer and the generator supplies the estimated transfer function. This arangement can be an alternative of the FFTbased analysis since it works as a filter bank and provides the measurement results on-line.

This work was supported by the Janos Bolyai Scolarship of the Hungarian Academy of Sciences and the Hungarian National Scientific Research Fund (OTKA) under contract number F 033055.

0-7803-6646-8/01/\$10.00 @2001 IEEE

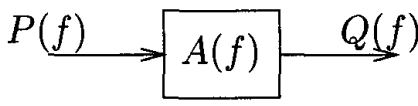

Fig. 1. The system to be identified

Section II. recalls the identification problem and the resonator based observer. Section III. introduces the resonator based identification and a detailed analysis is given. Real measurement data are provided in section IV., while section V. is the conlusion.

\section{PRELIMINARIES}

A. Non-parametric frequency domain identification of linear systems

Let $A(f)$ be a linear, time-invariant discrete time system as it is depicted in Fig. 1, where $P(f)$ and $Q(f)$ denote the Fourier transform of the input and the output signal, respectively. The non-parametric frequency domain identification of $A(f)$ is the estimation of its samples over a finite set of $f_{k}$ [1]:

$$
\hat{A}\left(f_{k}\right)=\frac{P\left(f_{k}\right)}{Q\left(f_{k}\right)}
$$

where $\hat{A}\left(f_{k}\right)$ is the estimation of $A\left(f_{k}\right)$. There are many possibilities to excite the system, a possible choice is the utilization of multisine excitation [3]. In this case $Q\left(f_{k}\right)$ is usually known in advance, and $P\left(f_{k}\right)$ is calculated by the DFT. If the length of the input sequence equals to the number of the DFT points, the estimation is not distorted due to the leakage and the picket fence effect. There are different averaging technics to reduce the random noise corrupting the measurement. If the identification should follow the changes in $A(f)$, exponential averaging can be used.

\section{$B$. The resonator based observer}

The resonator based observer was designed to follow the state variables of the so-called conceptual signal model [5]. The signal model is described as follows:

$$
\begin{gathered}
y_{n}=\mathbf{c}_{n}^{T} \mathbf{x}_{n} \\
\mathbf{c}_{n}=\left[c_{n, k}\right]=e^{j 2 \pi f_{k} n}, k=-L \ldots L
\end{gathered}
$$




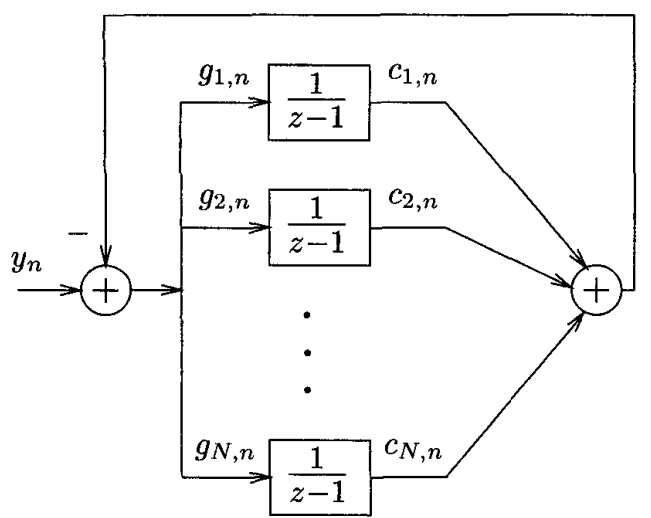

Fig. 2. The resonator based observer

$$
f_{-k}=-f_{k}, f_{0}=0, k=-L \ldots L
$$

where $\mathbf{x}_{n}$ is the state vector of the signal model at time step $n$, $y_{n}$ is its output (the input of the observer), $c_{n}$ represents the basis functions. Eq. (4) ensures that the model generates a real signal. This restriction is not necessary, but advantegeous in most cases. The conceptual signal model can be considered as a summed output of resonators which can generate any multisine with components up to the half of the sampling frequency. The corresponding observer is (Fig. 2):

$$
\hat{\mathbf{x}}_{n+1}=\hat{\mathbf{x}}_{n}+\mathbf{g}_{n}\left(y_{n}-\mathbf{c}_{n}^{T} \hat{\mathbf{x}}_{n}\right) ; \quad \mathbf{g}_{n}=\left[g_{n, k}\right]=r_{k} \bar{c}_{n, k}
$$

where $\hat{\mathbf{x}}_{n}$ is the estimated state vector, $\left\{r_{k} ; k=1 . . N ; N=\right.$ $2 L+1\}$ are free parameters to set the poles of the system, and the overbar denotes the complex conjugate operator. Due to the complex exponentials, the channels of the observer can be considered as time-invariant systems with a pole on the unit circle. This is why they are called resonators. Each channel of the observer is a filter output, the transfer functions of which is:

$$
H_{k}(z)=\frac{\frac{r_{k} z_{k}}{z-z_{k}}}{1+\sum_{i=1}^{N} \frac{r_{i} z_{i}}{z-z_{i}}}, k=1 \ldots N
$$

where $\left\{z_{k} ; k=1 . . N\right\}$ are the resonator poles. If the resonator poles are arranged uniformly on the unit circle, and $\left\{r_{k}=1 / N ; k=1 . . N\right\}$, the observer has finite impulse response, and the observer corresponds to the RDFT [5]. In that case the transfer function (6) is very simple:

$$
H_{k}(z)=\frac{1}{N} \frac{z^{N}-1}{z-z_{k}} z^{-N}, k=1 \ldots N
$$

the magnitude response of which is:

$$
\left|U_{k}(f)\right|=\left|\frac{\sin \pi N\left(f-f_{k}\right)}{N \sin \pi\left(f-f_{k}\right)}\right|, k=1 \ldots N
$$

(8) has zeros at each resonator frequency, except when $f=f_{k}$, where $U_{k}(f)=1$.
In practical applications where the fundamental frequency changes, the resonators cannot be placed uniformly, and the above setting of parameters $r_{k}$ does not provide finite impulse response. The adaptive Fourier Analyzer described in [6] adapts the resonator frequencies to coincide with those of the input signal, avoiding the picket-fence effect and leakage. It was successfully utilized e.g. in high-precision vectorvoltmeters [6] or in active noise control systems [7].

\section{RESONATOR BASED IDENTIFICATION}

\section{A. The identification system}

The above described resonator based generator - observer pair can be used for frequency domain non-parametric system identification as it is depicted in Fig. 3. The excitation is given by the state vector of the generator $\left(\mathbf{x}_{0}\right)$ which does not change while the identification is in progress. The system to be identified $(A(z))$ has to be in between the generator and the observer and the ratio of the corresponding state variables of the observer and the generator supply the results:

$$
\hat{A}\left(f_{k}\right)=\frac{\hat{x}_{k}}{x_{k}}, k=1 \ldots N
$$

Exponential averaging is an option of the structure, and it is controlled by the parameter $\alpha$. Its role is discussed in detail in the following subsections.

Since the same basis functions $\mathbf{c}_{n}$ are applied both in the generator and the observer, no picket fence effect and leakage occurs, even if finite wordlength effects are taken into consideration. The operation of the method can be characterized by noise suppression and measurement time. These are discussed below.

\section{B. Identification over a uniform resonator set}

The resonators are arranged uniformly on the unit circle, if $f_{1}=1 / N$. If $\left\{r_{k}=1 / N ; k=1 . . N\right\}$, the observer performs the RDFT [5]. It is the case when $\alpha=1$ in Fig. 3. Each channel has an equivalent noise bandwidth of $1 / N$. If the measurement noise is white, the ratio of the variances are:

$$
\frac{\sigma_{1}^{2}}{\sigma_{0}^{2}}=\frac{1}{N}
$$

where $\sigma_{0}^{2}$ is the variance of the original measurement noise, and $\sigma_{1}^{2}$ is the variance of the output. The system has finite impulse response, and the measurement time is $N$ steps.

If $0<\alpha<1$, the measurement results are exponentially averaged. The equivalent time constant is:

$$
\beta=1-(1-\alpha)^{1 / N}
$$




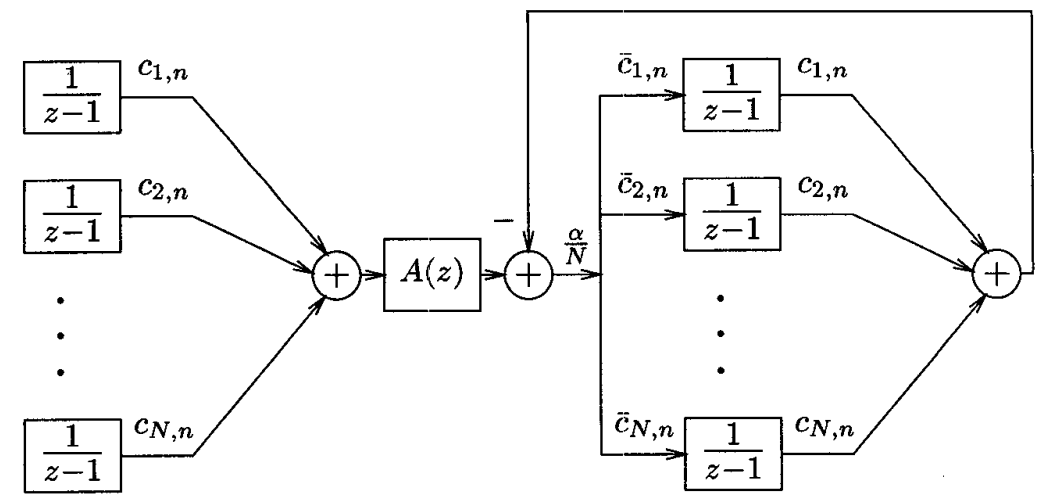

Fig. 3. Resonator based identification

This averaging improves the noise suppression of (10) (see e.g. [8]):

$$
\frac{\sigma_{2}^{2}}{\sigma_{0}^{2}} \approx \frac{\beta}{2}
$$

where $\sigma_{0}^{2}$ is the variance of the original measurement noise, and $\sigma_{2}^{2}$ is the variance of the averaged output. Since the system has infinite impulse response, the measurement time depends on the accuracy of the measurement:

$$
K \approx \frac{\log \varepsilon}{\log (1-\beta)}
$$

where $\varepsilon$ denotes the final error to be achieved. Note that in practical cases first $\beta$ is determined upon the specification of the identification task, and $\alpha$ is calculated by the inverse of (11):

$$
\alpha=1-(1-\beta)^{N}
$$

\section{Identification over an arbitrary resonator set}

In many practical cases the identification shall be done over a non-uniform frequency set: e.g. acoustical measurements require logarithmic frequency points. In these cases (7) and (8) are no more valid, and the system has infinite impulse response, even if $\alpha=1$. However, it remains that the $k$-th transfer function has zeros at each resonator frequency, except when $f=f_{k}$, where it is exactly 1 . It means that the structure is able to perform undistorted measurements, according to (9). Note that the identification in this case does not require extra calculations compared to the uniform resonator set case.

The calculation of the noise suppression and the measurement time is generally very complicated, since each channel has different equivalent noise bandwidth. Fortunately, if $\beta \ll 1$, the relevant transfer functions can be well approximated as follows:

$$
H_{k}(z)=\frac{\frac{r_{k} z_{k}}{z-z_{k}}}{1+\frac{r_{k} z_{k}}{z-z_{k}}+\sum_{i=1, i \neq k}^{N} \frac{r_{i} z_{i}}{z-z_{i}}} \approx
$$

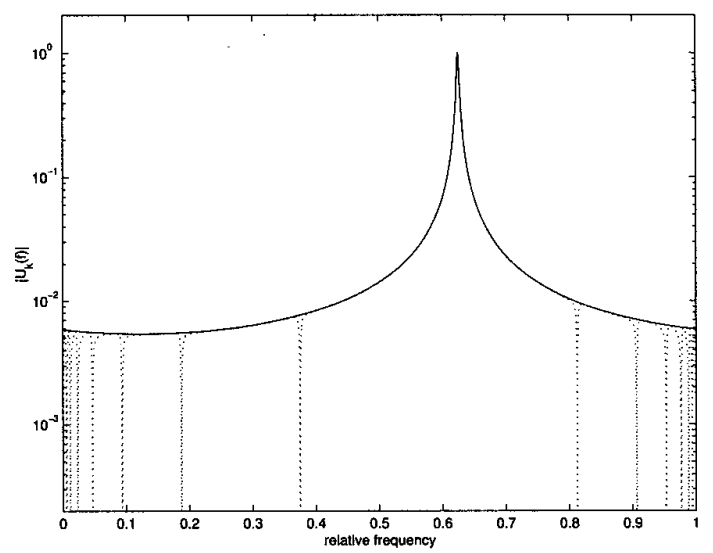

Fig. 4. Magnitude response of one resonator channel with logarithmic frequency set $(\beta=0.01$ ). Solid line: magnitude response of the approximating structure; dashed line: magnitude response of the original structure

$$
\approx \frac{\frac{r_{k} z_{k}}{z-z_{k}}}{1+\frac{r_{k} z_{k}}{z-z_{k}}}=\frac{r_{k} z_{k}}{z-z_{k}\left(1-r_{k}\right)}, k=1 \ldots N
$$

In this case the $k$-th channel can be approximated with another resonator based observer output, which contains one resonator only, at the frequency of $f_{k}$, with $r_{k}=\beta$. The approximation is good along the frequency axis, with the exception of the neighborhood of the original resonator positions, since the approximating transfer function has no zeros at those places. This is demonstrated in Fig. 4. The figure shows that the magnitude response of the original and the associated structure are close to each other. It also implies that the equivalent noise bandwidth is nearly the same for the two structures, so (12) is a good estimation for the case of an arbitrary resonator set.

Due to the Parseval's theorem, the measurement time can be estimated by the corresponding transfer functions. The squared error of the approximating transfer function (15) tends to zero as $\beta$ tends also to zero. Numerical simulations show that the error of the approximation can be omitted for $\beta$ values frequently 


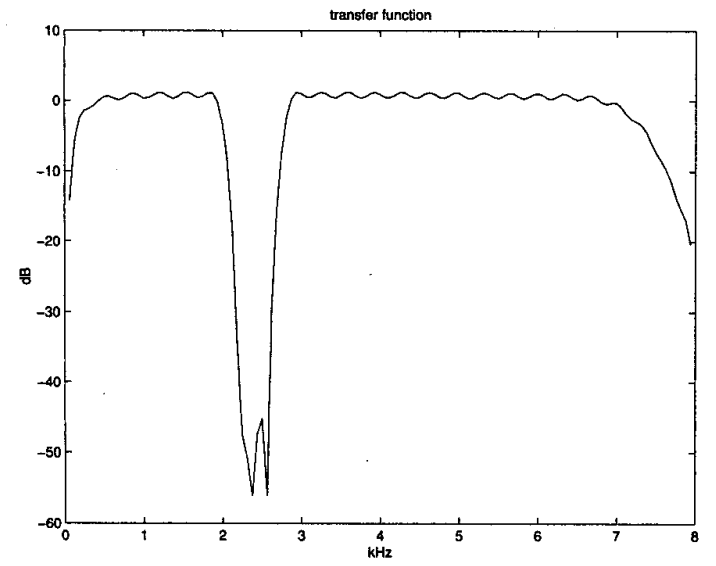

Fig. 5. Result of the identification of a band-stop FIR filter.

used in the practice $(\beta \leq 0.01)$. Thus $(13)$ is a good estimation also for the measurement time in the case of an arbitrary resonator set.

\section{MEASUREMENTS}

In order to test the algorithm, a simple DSP program was written. The proposed identification method was implemented on an ADSP 2181 based EZ-KIT Lite development board [9]. (ADSP 2181 is a 16-bit fixed-point signal processor [10].) It has two analog channels with 16-bit delta-sigma A/D and D/A converters. The sampling frequency can be set from 5.5125 $\mathrm{kHz}$ to $48 \mathrm{kHz}$, in several steps. The system to be identified has to be connected in between the D/A and the A/D converters. The input variables of the program are: the multisine excitation sequence (thus the number of the resonators), and the time constant $\beta$ for exponential averaging (see Section III.). The proposed identification is an on-line method, so the resolution is limited by the computational complexity of the method. Up to the sampling frequency of $8 \mathrm{kHz}$, the transfer function can be measured in 512 points. At the maximal sampling frequency of $48 \mathrm{kHz}$, the measurement can be done in 64 points.

Now the proposed method is illustrated by the identification of a band-stop filter. It is a finite impulse response (FIR) filter implemented on another DSP board, with a sampling frequency of $16 \mathrm{kHz}$. The measurement result can be seen in Fig. 5. The transfer function is measured in 256 point, i.e. with a resolution of $62.5 \mathrm{~Hz}$. Damping near to $\mathrm{DC}$ and $8 \mathrm{kHz}$ are due to the $\mathrm{AC}$ coupling and the delta-sigma $\mathrm{A} / \mathrm{D}$ and $\mathrm{D} / \mathrm{A}$ converters. The suppression in the stop band is about $50 \mathrm{~dB}$, while the specification is $60 \mathrm{~dB}$. This difference is because of the 16-bit wordlength.

The settling of the system can be seen in Fig. 6. The figure shows the feedback error of the structure (see Fig. 2) in the case of the above measurement, with a time constant of $\beta=0.005$. The settling can be considered complete if this error signal is

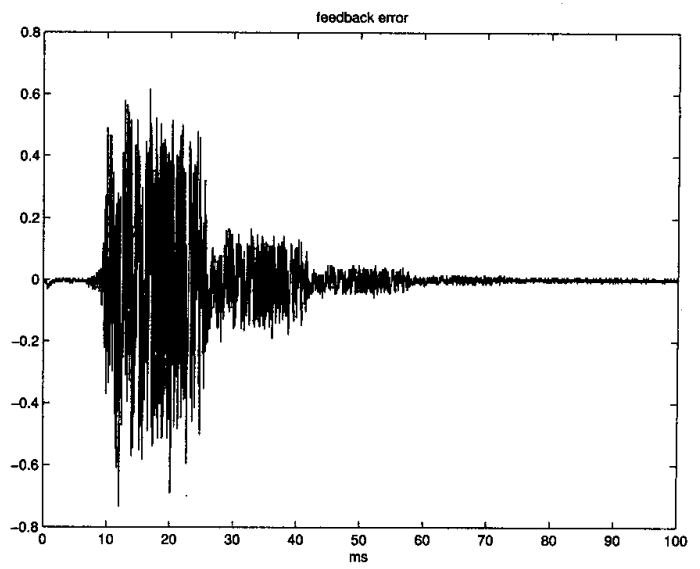

Fig. 6. The settling of the resonator based structure in the case of time constant $\beta=0.005$.

zero. Although the settling of the system is exponential, the decay is not continuous: the magnitude of the transient changes only in every $16 \mathrm{~ms}$ (in every $N=256$ steps), when it is multiplied by $\alpha$. In the example $\alpha \approx 0.28$, according to (14). This settling is characteristic only when the resonators are arranged uniformly.

\section{CONCLUSION}

A nonparametric identification method for linear systems was introduced. The paper described the theoretical background of the method and it was illustrated by a practical example. The advantage of the proposed structure is that it works as a filter bank and provides the measurement results on-line, while the feedback-based observer ensures high-precision measurements. The identification can be performed over any frequency set, e.g. over a logarithmic set. The computational demand of the method depends only on the number of the frequency points. The method is an alternative of the FFT-based analysis where changes in a plant should be followed.

\section{REFERENCES}

[1] L. Ljung, "System Identification. Theory for the User", Prentice-Hall, Inc. 1999.

[2] J. Schoukens, R. Pintelon, "Identification of Linear Systems", Pergamon Press 1991.

[3] K. Godfrey (ed.), "Perturbation Signals for System Identification", Prentice-Hall, Inc. 1993.

[4] G. H. Hostetter, "Recursive discrete Fourier transformation" IEEE Trans. Acoust., Speech, Signal Processing Vol. ASSP-28 No. 2. pp. 183-190, Apr. 1980.

[5] G. Péceli, "A common structure for recursive discrete transforms", IEEE Trans. Circuits Syst. Vol. CAS-33 No. 10. pp. 1035-36, Oct. 1986.

[6] F. Nagy, "Measurement of signal parameters using nonlinear observers", IEEE Trans. Instrum. Meas., Vol. IM-41 No. 1. pp. 152-155, Feb. 1992.

[7] L. Sujbert, G. Péceli, "Periodic noise cancelation using resonator based controller", in 1997 Int Symp. on Active Control of Sound and Vibration, ACTIVE' '97, pp. 905-916, Budapest, Hungary, Aug. 1997.

[8] L. Schnell (ed.), "Technology of Electrical Measurements", Wiley, 1993.

[9] ADSP-2100 EZ-KIT Lite Reference Manual, Analog Devices, Inc., 1995.

[10] ADSP-2100 Family User's Manual, Analog Devices, Inc., 1995. 